\title{
Coronary calcium score as a predictor of outcomes in the hypertensive Covid-19 population: results from the Italian (S) Core- Covid-19 Registry
}

\author{
Alberto Cereda $^{1,2} \cdot$ Marco Toselli $^{1} \cdot$ Anna Palmisano ${ }^{3,4} \cdot$ Davide Vignale $^{3,4} \cdot$ Arif Khokhar $^{1} \cdot$ Gianluca Campo $^{5}$. \\ Matteo Bertini ${ }^{5}$. Marco Loffi ${ }^{6}$ - Daniele Andreini ${ }^{7}$ Gianluca Pontone ${ }^{7}$. Gianluigi Patelli ${ }^{8}$. \\ Giuseppe Massimo Sangiorgi ${ }^{9} \cdot$ Gabriele Tumminello $^{11} \cdot$ Mariarosa Elia $^{2} \cdot$ Attilio lacovoni $^{10} \cdot$ Stefano Carugo $^{11}$. \\ Claudio Rapezzi $^{1,5}$ - Antonio Colombo ${ }^{1}$ - Francesco Giannini ${ }^{1}$ - Antonio Esposito ${ }^{3,4}$
}

Received: 14 August 2021 / Revised: 25 September 2021 / Accepted: 28 September 2021 / Published online: 18 November 2021

(c) The Japanese Society of Hypertension 2021

\begin{abstract}
Hypertension is associated with more severe disease and adverse outcomes in COVID-19 patients. Recent investigations have indicated that hypertension might be an independent predictor of outcomes in COVID-19 patients regardless of other cardiovascular and noncardiovascular comorbidities. We explored the significance of coronary calcifications in 694 hypertensive patients in the Score-COVID registry, an Italian multicenter study conducted during the first pandemic wave in the Western world (March-April 2020). A total of 1565 patients admitted with RNA-PCR-positive nasopharyngeal swabs and chest computed tomography (CT) at hospital admission were included in the study. Clinical outcomes and cardiovascular calcifications were analyzed independently by a research core lab. Hypertensive patients had a different risk profile than nonhypertensive patients, with more cardiovascular comorbidities. The deceased hypertensive patients had a greater coronary calcification burden at the level of the anterior descending coronary artery. Hypertension status and the severity cutoffs of coronary calcifications were used to stratify the clinical outcomes. For every $100-\mathrm{mm}^{3}$ increase in coronary calcium volume, hospital mortality in hypertensive patients increased by $8 \%$, regardless of sex, age, diabetes, creatinine, and lung interstitial involvement. The coronary calcium score contributes to stratifying the risk of complications in COVID-19 patients. Cardiovascular calcifications appear to be a promising imaging marker for providing pathophysiological insight into cardiovascular risk factors and COVID-19 outcomes.
\end{abstract}

Keywords Hypertension · Covid-19 · Cardiovascular Calcification • Coronary Calcification • Calcium Score

\section{Introduction}

Hypertension is the most common primary modifiable risk factor associated with atherosclerotic cardiovascular disease

Alberto Cereda

alberto.cereda@email.it

GVM Care \& Research Maria Cecilia Hospital, Cotignola, Italy

ASST Santi Paolo e Carlo, Milan, Italy

IRCCS San Raffaele Scientific Institute, Milan, Italy

Vita-Salute San Raffaele University, Milan, Italy

Azienda Ospedaliero-Universitaria di Ferrara, Cona, FE, Italy
[1]. Its prevalence is closely related to age, and it is estimated to affect $\sim 50 \%$ of the population over 60 years of age.

In the current COVID-19 pandemic, hypertension has been identified as a common cardiovascular risk factor in
6 Ospedale di Cremona, Cremona, Italy

7 Centro Cardiologico Monzino IRCCS, Milan, Italy

8 ASST Bolognini Hospital, Bergamo Est, Italy

9 Università degli Studi di Roma "Tor Vergata", Roma, Italy

10 ASST Papa Giovanni XXIII, Bergamo, Italy

11 Fondazione IRCCS Cà Granda Ospedale Maggiore Policlinico, Division of Cardiology, Department of Clinical Sciences and Community Health, University of Milan, Milan, Italy 
hospitalized patients and is highly associated with COVID19 disease severity and adverse outcomes.

This finding may reflect the clinical profile of patients admitted with COVID-19 infection, who are often older, male, and have multiple cardiovascular comorbidities, all known risk factors for developing more severe SARS-CoV2 infection [2-5].

However, whether hypertension itself is an independent predictor of COVID-19 severity and mortality remains unknown, as does the potential pathophysiological link.

From this perspective, chest CT can help us to evaluate the extent of viral pneumonia and allows us to quantify and qualify cardiovascular calcifications, a surrogate marker of cardiovascular disease.

Chest CT was usually performed during the COVID-19 pandemic for pneumonia diagnosis, assessment of severity, and prognostication.

Behind the assessment of lung involvement, several features of cardiovascular risk can be extracted from chest CT [6-11], providing a more comprehensive characterization of the risk profile of infected patients. In particular, the coronary calcium score, easily measured from chest CT scans, is a highly specific marker of coronary atherosclerosis and an established cardiovascular prognosticator [6, 8-11].

However, the higher mortality observed in hypertensive COVID-19 patients than in nonhypertensive patients could be explained by age, male sex, higher prevalence of diabetes, and chronic renal failure.

Statistical collinearity between age, hypertension and other risk factors limits the assumptions of multivariable regression analyses, leaving the issue unsolved.

Coronary artery calcium (CAC) is a specific marker of atherosclerosis burden that can be easily measured from nongated chest computed tomography (CT), which is often performed for the assessment of SARS-CoV-2 pneumonia. Its predictive significance has not yet been specifically investigated in the hypertensive COVID-19 population.

Coronary artery calcium (CAC) and thoracic aortic calcium assessed by chest computed tomography (CT) were also predictors of death in patients admitted due to COVID19 undergoing chest CT for assessment of pneumonia [8].

Imaging biomarkers such as coronary artery calcium (CAC) score have an established role in long-term cardiovascular event risk stratification but might also provide important prognostic information and pathophysiologic insights in patients acutely ill with COVID-19.

The use of CT affords the opportunity to noninvasively construct cross-sectional images of arteries to detect the presence and extent of calcium attributed to atherosclerosis in different vascular beds.

Many risk factors, including hypertension, are associated with atherosclerotic calcification.
It has been shown that vascular calcification is associated with increased arterial stiffness [9].

In normotensive subjects, CAC was associated with retinopathy, suggesting a common pathophysiologic process underlying both micro- and macrovascular disease. Several studies have shown a clear association between hypertension and CAC, which are both independently related to systemic arterial abnormalities $[10,11]$.

\section{Study aims}

The present study aims to explore the prognostic significance of coronary calcium in a large cohort of COVID19 hypertensive patients.

\section{Methods}

Consecutive patients with positive RT-PCR for SARSCoV-2 and a noncontrast chest CT performed at hospital admission from March 1st to April 20th, 2020 in 16 Italian hospitals were included in the study.

The exclusion criteria were previous coronary artery stenting for impaired quantitative measurement of calcium score.

The study was approved by the regional ethics committee of the Emilia Romagna region and by the ethics committee of the individual hospitals.

The following clinical and laboratory variables were derived from electronic medical records from each center: demographic characteristics (age, sex, and body mass index), cardiovascular risk factors (hypertension, coronary heart disease, diabetes, peripheral artery disease), comorbidities (chronic lung disease, chronic kidney disease, active neoplasms), laboratory data at admission (hemoglobin, white blood cell count, creatinine), lactate dehydrogenase (LDH), C-reactive protein (CRP), and patient outcomes (inhospital death, intubation, noninvasive ventilation, oxygen therapy).

\section{Chest CT data}

Chest CTs were collected and analyzed in a single Core Lab, where images were reformatted at a standard slice thickness of $2.5 \mathrm{~mm}$ without overlap or gaps and visualized using a standard mediastinal window (width $350 \mathrm{HU}$; center $40 \mathrm{HU})$.

The coronary calcium score and calcium volume were semiautomatically extracted by expert cardiac radiologists blinded to clinical data using dedicated software 
(Intellispace Portal v. 8.0, Philips). In addition, a qualitative assessment of coronary artery calcification was performed.

Pneumonia lung involvement was assessed semiquantitatively as follows: score 0 : absent; score 1: $<25 \%$, score 2: $25-50 \%$, score 3: 50-75\%, and score $4:>75 \%$ [7].

\section{Statistical analysis}

Continuous and categorical variables are displayed as the mean \pm standard deviation (SD) and percentage, respectively.

Student's $t$ test was used to assess the differences between continuous data. Differences were analyzed by the chi-square test for categorical variables and analysis of variance (ANOVA) for continuous variables.

The population was classified into eight groups based on the presence or absence of hypertension and the severity of coronary calcium calcifications using established cutoffs in the radiological literature (CAC 0: absent; 0-100: mild; 100-400: moderate and $\mathrm{CAC}>400$ for severe coronary calcification burden).

The coronary calcium volume variable was then categorized as $>100 \mathrm{~mm}^{3}$ and $>400 \mathrm{~mm}^{3}$ in the respective Cox regression models for mortality. Therefore, a model categorizing $100-\mathrm{mm}^{3}$ incremental values of coronary calcium volume was applied. The results are reported as hazard ratios (HRs) with associated $95 \%$ confidence intervals (CIs).

The mortality prediction of coronary calcium (both as a continuous and categorical variable) in hypertensive patients was tested with Cox proportional hazards models for age, sex, creatinine, diabetes, and lung interstitial involvement variables.

The results were considered statistically significant when the $P$ value was $<0.05$.

Statistical analysis was performed using SPSS (version 23, Chicago, IL, USA).

\section{Results}

\section{Characteristics of hypertensive and nonhypertensive patients}

The final cohort of 1561 patients consisted mainly of men $(1043 / 1565,66.6 \%)$, with a mean age of $68 \pm 13$ years. Most of the patients suffered from diabetes (297/1565, $19 \%)$, as well as ischemic heart disease $(177 / 1565,11.3 \%)$ and chronic lung disease $(164 / 1565,10.5 \%)$ (Table 1).

At admission, patients had hemoglobin values in the normal range, with a mean creatinine of $1.19 \pm 0.76 \mathrm{mg} / \mathrm{dL}$.

Most of the patients $(674 / 1565,43.3 \%)$ had moderate pneumonia extension at hospital admission. The median coronary calcium volume was $34 \mathrm{~mm}^{3}\left(0-263 \mathrm{~mm}^{3}\right)$.
Orotracheal intubation occurred in $19.3 \%$ of patients, and $22.7 \%$ of patients died.

Compared with nonhypertensive patients, hypertensive patients were older $(72.3 \pm 11$ vs. $62.3 \pm 14, p$ value 0.001$)$ and had a similar sex distribution (233/694 in hypertensive vs. $289 / 871$ in nonhypertensive patients, $p$ value 0.85 ).

Diabetes was three times more frequent in hypertensive patients, while coronary heart disease was $\sim 4$ times more frequent than in nonhypertensive patients. Hypertensive patients did not differ from nonhypertensive patients in regard to white blood cells or CRP and had slightly higher creatinine values $(1.25 \pm 0.75$ vs. $1.13 \pm 0.8, p$ value 0.001$)$ and higher $\mathrm{LDH}$ values.

Pneumonia on chest CT was not significantly different between hypertensive and nonhypertensive patients.

In addition, concerning cardiovascular calcifications, the median values of coronary, aortic valvular, thoracic aortic, and left anterior descending calcium volumes did not differ significantly. There were no significant differences in outcomes other than mortality, which was higher in hypertensive patients (15.2 vs. $7.5 \%, p$ value 0.001$)$ (Table 1).

\section{Hypertensive patients: survivors vs. nonsurvivors}

The deceased hypertensive patients were older (77.9 \pm 9 vs. $70 \pm 11$ years old, $p$ value 0.001 ), with a higher prevalence of men $(93.5 \%$ vs. $73 \%, p$ value 0.001$)$ and compromised renal function $(1.6 \pm 1$ vs. $1.1 \pm 0.5 \mathrm{mg} / \mathrm{dl}, p$ value 0.001$)$. Deceased patients had severe systemic inflammation documented by higher values of white blood cells and CRP (WBC: $8260 \pm 4000$ vs. $7500 \pm 3720 / \mathrm{mm}^{3}, p$ value 0,01 ; CRP: $14 \pm 9$ vs. $11 \pm 9, p$ value 0,001$)$ and slightly worse baseline oxygen saturation $(87 \pm 9$ vs. $90 \pm 11 \%, p$ value 0.001).

Cardiovascular comorbidities, unexpectedly, were not more frequent in deceased hypertensive patients who had a greater calcium burden in the anterior descending artery (CAC - LAD: $17 \mathrm{~mm}^{3}$ vs. $13.5 \mathrm{~mm}^{3}$ in survivors, $p$ value 0.027).

Acute coronary syndromes and strokes were slightly more frequent in deceased hypertensive patients (stroke: $1.1 \%$ vs. $0.8 \%, p$ value 0.008 ; acute coronary syndrome: $0.5 \%$ vs. $0.4 \%, p$ value 0.071$)$. There were no significant differences regarding pulmoiary embolism (Tables 2, 3).

\section{Hypertension and coronary calcium burden severity}

Based on the presence of hypertension and calcium score classes, the population was classified into eight groups that were significantly different in terms of age, creatinine, pneumonia severity, intubation rate, stroke rate, and mortality. 
Table 1 Demographic and clinical variables

\begin{tabular}{|c|c|c|c|c|}
\hline Variables & $\begin{array}{l}\text { Hypertensive } \\
\text { patients }\end{array}$ & $\begin{array}{l}\text { Not hypertensive } \\
\text { patients }\end{array}$ & All patients & $P$ value \\
\hline Number of patients, $N(\%)$ & $871(7,55)$ & $694(3,44)$ & $1565(100)$ & \\
\hline Age, years, \pm SD & $72.3 \pm 11$ & $62.3 \pm 14$ & $67.9 \pm 13$ & 0.001 \\
\hline Female sex, $n / N(\%)$ & $289(5,18)$ & $233(9,14)$ & $522(4,33)$ & 0.85 \\
\hline Type 2 diabetes mellitus, $n / N(\%)$ & $226(14,4)$ & $71(4,5)$ & $297(19)$ & 0.001 \\
\hline History of CAD, $n / N(\%)$ & $141(9)$ & $36(2,3)$ & $177(11,3)$ & 0.001 \\
\hline History of Atrial Fibrillation, $n / N(\%)$ & $114(7,5)$ & $26(1,7)$ & $140(9,3)$ & 0.001 \\
\hline History of PAD, $n / N(\%)$ & $71(4,5)$ & $20(1,3)$ & $91(5,8)$ & 0.001 \\
\hline History of smoking, $n / N(\%)$ & $50(4,2)$ & $15(1,3)$ & $65(4,2)$ & 0.001 \\
\hline Chronic lung disease, $n / N(\%)$ & $116(7,4)$ & $48(3,1)$ & $164(10,5)$ & 0.001 \\
\hline Previous oncological malignancy, $n / N(\%)$ & $43(2,7)$ & $34(2,2)$ & $77(4,9)$ & 0.97 \\
\hline \multicolumn{5}{|l|}{ Laboratory variables } \\
\hline Hemoglobin, $\mathrm{g} / \mathrm{dl} \pm \mathrm{SD}$ & $13.2 \pm 2$ & $13.6 \pm 1.9$ & $13.3 \pm 2$ & 0.001 \\
\hline Creatinine, $\mathrm{mg} / \mathrm{dl} \pm \mathrm{SD}$ & $1.25 \pm 0.75$ & $1.13 \pm 0.8$ & $1.19 \pm 0.76$ & 0.001 \\
\hline White blood cells, $n / 1000 \pm \mathrm{SD}$ & $7720 \pm 3812$ & $7710 \pm 4322$ & $7715 \pm 4040$ & 0.96 \\
\hline C-Reactive protein, $\mathrm{mg} / \mathrm{dl} \pm \mathrm{SD}$ & $12.0 \pm 9.1$ & $12.3 \pm 9.8$ & $12 \pm 9.4$ & 0.44 \\
\hline $\mathrm{LDH}, \mathrm{u} / 1 \pm \mathrm{SD}$ & $405 \pm 239$ & $373 \pm 255$ & $392 \pm 246$ & 0.035 \\
\hline \multicolumn{5}{|l|}{ Respiratory variables } \\
\hline Baseline on air oxygen saturation, $n \pm \mathrm{SD}$ & $89 \pm 10$ & $89 \pm 13$ & $89 \pm 12$ & 0.63 \\
\hline $\begin{array}{l}\text { Lung CT interstitial involvement }<25 \% \text {, } \\
n / N(\%)\end{array}$ & $270(17,3)$ & $231(14,8)$ & $501(32)$ & 0.33 \\
\hline $\begin{array}{l}\text { Lung CT interstitial involvement } \\
25-50 \%, n / N(\%)\end{array}$ & $384(44,6)$ & $290(18,5)$ & $674(43,1)$ & 0.36 \\
\hline $\begin{array}{l}\text { Lung CT interstitial involvement } \\
50-75 \%, n / N(\%)\end{array}$ & $177(11,3)$ & $128(8,2)$ & $305(19,5)$ & 0.35 \\
\hline $\begin{array}{l}\text { Lung CT interstitial involvement }>75 \% \text {, } \\
n / N(\%)\end{array}$ & $28(1,8)$ & $34(2,2)$ & $62(4)$ & 0.09 \\
\hline \multicolumn{5}{|l|}{ Cardio-thoracic vascular calcifications } \\
\hline Coronary calcium volume, $\mathrm{mm}^{3}$ & $34.3(0-283)$ & $34(0-253)$ & $34(0-263)$ & 0.91 \\
\hline LAD coronary calcium volume, $\mathrm{mm}^{3}$ & $15(0-138)$ & $14(0-130)$ & $14(0-134)$ & 0.98 \\
\hline Thoracic aorta calcium volume, $\mathrm{mm}^{3}$ & $383(19-2461)$ & $302(14-2153)$ & $362(16-2304)$ & 0.22 \\
\hline Aortic valve calcium volume, $\mathrm{mm}^{3}$ & $0(0-80)$ & $0(0-50)$ & $0(0-63)$ & 0.11 \\
\hline \multicolumn{5}{|l|}{ Clinical outcomes } \\
\hline $\begin{array}{l}\text { Respiratory support with non invasive } \\
\text { ventilation without intubation, } n / N(\%)\end{array}$ & $142(9,1)$ & $117(7,5)$ & $259(16,5)$ & 0.76 \\
\hline Orotracheal intubation, $n / N(\%)$ & $163(10.6 \%)$ & $134(8,7)$ & $297(19,3)$ & 0.74 \\
\hline Pulmonary embolism, $n / N(\%)$ & $258(1,6)$ & $28(1,8)$ & $53(3,4)$ & 0.2 \\
\hline Acute coronary syndrome, $n / N(\%)$ & $7(0,5)$ & $8(0,5)$ & $15(1)$ & 0.48 \\
\hline Stroke, $n / N(\%)$ & $16(1)$ & $9(0,6)$ & $25(1,6)$ & 0.39 \\
\hline Time to death after admission, $n \pm \mathrm{SD}$ & $9 \pm 7$ & $8 \pm 6$ & $9 \pm 6$ & 0.25 \\
\hline Death, $n / N(\%)$ & $238(15,2)$ & $117(7,5)$ & $355(22,7)$ & 0.001 \\
\hline
\end{tabular}

Descriptive table with the characteristics of the entire population, of hypertensive and non-hypertensive patients

Patients without hypertension and with calcium scores of zero were on average 10 years younger than patients in other groups.

The mortality rate and oxygen therapy differed among the 8 groups (Figs. 1, 2).

In particular, Fig. 3 shows the risk of hospital death with a colorimetric map (risk $<2 \%$, risk $2-5 \%$, risk $>5 \%$ ).
The risk of hospital death was especially increased in patients with $\mathrm{CAC}>400 \mathrm{~mm}^{3}$. In patients with $\mathrm{CAC}<$ $400 \mathrm{~mm}^{3}$, nonhypertensive patients had a mortality rate $<2 \%$, in contrast to hypertensive patients $(2-5 \%$ risk of death).

The nonhypertensive patients with a calcium score of zero had a mortality rate of $1.6 \%$ compared to $7.5 \%$ in 
Table 2 Differences between survived and deceased hypertensive patients

\begin{tabular}{|c|c|c|c|}
\hline Variables & $\begin{array}{l}\text { Deceased hypertensive } \\
\text { patients }\end{array}$ & $\begin{array}{l}\text { Survived hypertensive } \\
\text { patients }\end{array}$ & $P$ value \\
\hline \multicolumn{4}{|l|}{ Demographic and clinical variables } \\
\hline Number of patients, $n / N(\%)$ & $238(27,3)$ & $633(72,6)$ & \\
\hline Age, years, \pm SD & $77.9 \pm 9$ & $70 \pm 11$ & 0.001 \\
\hline Sex, $n / N(\%)$ & $56(6,5)$ & $231(27)$ & 0.001 \\
\hline Type 2 diabetes mellitus, $n / N(\%)$ & $75(8,8)$ & $148(17,3)$ & 0.009 \\
\hline History of CAD, $n / N(\%)$ & $49(5,7)$ & $90(10,5)$ & 0.016 \\
\hline History of Atrial Fibrillation, $n / N(\%)$ & $44(5,2)$ & $67(7,9)$ & 0.001 \\
\hline History of PAD, $n / N(\%)$ & $32(3,7)$ & $39(4,6)$ & 0.001 \\
\hline History of smoking, $n / N(\%)$ & $14(2,3)$ & $36(6)$ & 0.49 \\
\hline Chronic lung disease, $n / N(\%)$ & $45(5,3)$ & $68(7,9)$ & 0.001 \\
\hline $\begin{array}{l}\text { Previous oncological malignancy, } \\
n / N(\%)\end{array}$ & $10(1,2)$ & $33(3,9)$ & 0.57 \\
\hline \multicolumn{4}{|l|}{ Laboratory values } \\
\hline Hemoglobin, g/dl \pm SD & $13 \pm 2$ & $13.3 \pm 2$ & 0.21 \\
\hline Creatinine, $\mathrm{mg} / \mathrm{dl} \pm \mathrm{SD}$ & $1.6 \pm 1$ & $1.1 \pm 0.5$ & 0.001 \\
\hline White blood cells, $n / 1000 \pm \mathrm{SD}$ & $8260 \pm 4000$ & $7500 \pm 3720$ & 0.011 \\
\hline C-Reactive protein, $\mathrm{mg} / \mathrm{dl} \pm \mathrm{SD}$ & $14 \pm 9$ & $11 \pm 9$ & 0.001 \\
\hline $\mathrm{LDH}, \mathrm{u} / \mathrm{l} \pm \mathrm{SD}$ & $496 \pm 279$ & $371 \pm 212$ & 0.001 \\
\hline \multicolumn{4}{|l|}{ Respiratory variables } \\
\hline $\begin{array}{l}\text { Baseline on air oxygen saturation, } \\
n \pm \mathrm{SD}(\%)\end{array}$ & $87 \pm 9$ & $90 \pm 11$ & 0.001 \\
\hline $\begin{array}{l}\text { Lung CT interstitial involvement } \\
<25 \%, n / N(\%)\end{array}$ & $70(8,2)$ & $195(22,8)$ & 0.81 \\
\hline $\begin{array}{l}\text { Lung CT interstitial involvement } \\
25-50 \%, n / N(\%)\end{array}$ & $98(11,4)$ & $281(32,8)$ & 0.51 \\
\hline $\begin{array}{l}\text { Lung CT interstitial involvement } \\
50-75 \%, n / N(\%)\end{array}$ & $52(6,1)$ & $121(14,1)$ & 0.303 \\
\hline $\begin{array}{l}\text { Lung CT interstitial involvement } \\
>75 \%, n / N(\%)\end{array}$ & $8(0,9)$ & $20(2,3)$ & 0.84 \\
\hline \multicolumn{4}{|l|}{ Cardio-thoracic vascular calcifications } \\
\hline Coronary calcium volume, $\mathrm{mm}^{3}$ & $44(0-426)$ & $31(0-247)$ & 0.1 \\
\hline LAD coronary calcium volume, $\mathrm{mm}^{3}$ & $17(0-211)$ & $13.5(0-119)$ & 0.027 \\
\hline Thoracic aorta calcium volume, $\mathrm{mm}^{3}$ & $432(15-2703)$ & $371(19-2366)$ & 0.36 \\
\hline Aortic valve calcium volume, $\mathrm{mm}^{3}$ & $0(0-57)$ & $0(0-85)$ & 0.35 \\
\hline \multicolumn{4}{|l|}{ Outcomes } \\
\hline Orotracheal intubation & $17(2)$ & $146(17)$ & 0.001 \\
\hline Pulmonary embolism, $n / N(\%)$ & $4(0,5)$ & $20(2,3)$ & 0.24 \\
\hline Acute coronary syndrome, $n / N(\%)$ & $4(0,5)$ & $3(0,4)$ & 0.071 \\
\hline Stroke, $n / N(\%)$ & $9(1,1)$ & $7(0,8)$ & 0.008 \\
\hline
\end{tabular}

Descriptive table with the characteristics of surviving and deceased hypertensive patients hypertensive patients with a calcium score $>400 \mathrm{~mm}^{3}$ ( $p$ value 0.001$)$ (Figs. 1, 4).

\section{Cox regression models for 30 -day mortality}

In univariate Cox regression, coronary calcium $\left(\mathrm{mm}^{3}\right)$ was a significant risk factor for mortality with an HR of 1.001 (95\% CI 1-1.001) and maintained its significance even in the multivariate model that included age, female sex, diabetes, creatinine, and pneumonia extension. A coronary calcium score $>100 \mathrm{~mm}^{3}$ was associated with mortality risk in the hypertensive population with an HR of 1.34 (95\% CI 1.029-1.757).

Similarly, a coronary calcium score greater than 400 $\mathrm{mm}^{3}$ was also associated with a comparable increased risk of mortality (HR 1.315 95\% CI 1.01-1.713). 


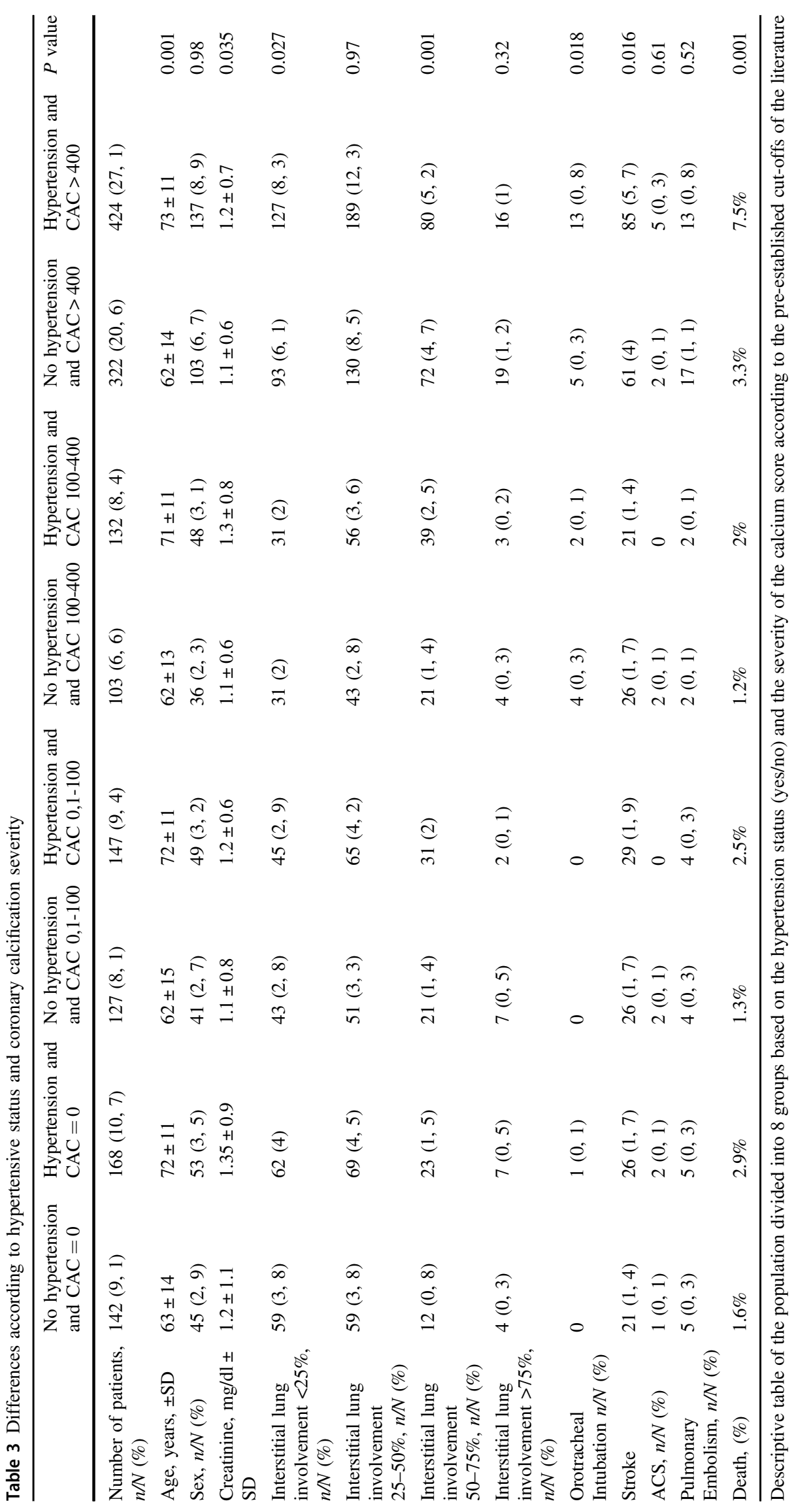


For every $100 \mathrm{~mm}^{3}$ increase in coronary calcium volume, there was an increase in mortality of approximately $8 \%$ (HR $1.08295 \%$ CI 1.033-1.133), regardless of age, sex, diabetes, creatinine, and pneumonia extension on CT.

In the Kaplan-Meier curves (Fig. 5), the survival curves of hypertensive patients with a coronary calcium score greater than $100 \mathrm{~mm}^{3}$ and greater than $400 \mathrm{~mm}^{3}$ diverge significantly. Death tended to occur increasingly earlier in patients with a greater coronary calcium burden (Fig. 5) (Table 4).

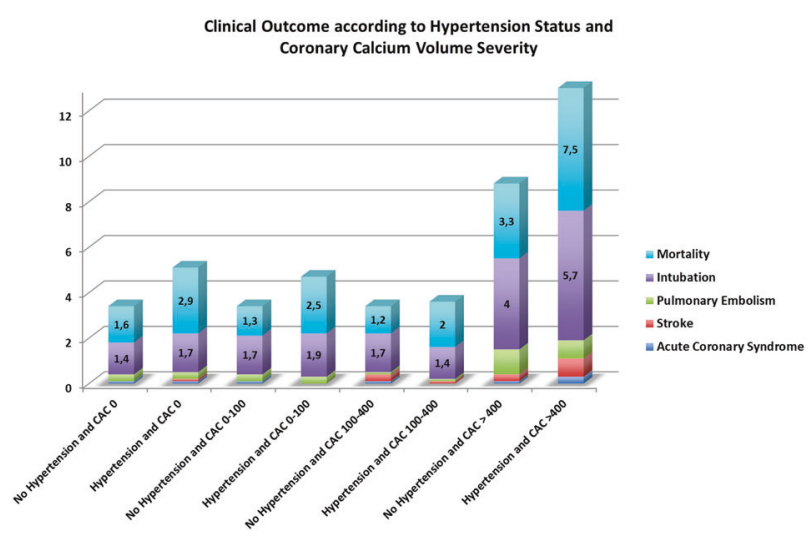

Fig. 1 Clinical outcomes

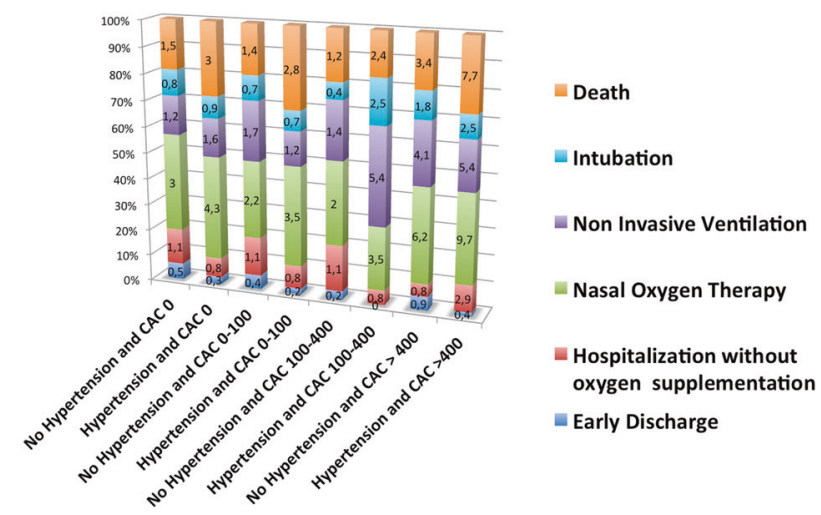

Fig. 2 Hospital and respiratory outcomes classified according to hypertensive status and coronary calcium volume

\section{Discussion}

Hypertension is a common risk factor, and in the literature, there is no real stratification of hypertensive patients based on known clinical and subclinical cardiac organ damage. Stratification based on anamnestic comorbidities in nonrandomized studies represents a limitation to the available studies.

Our results highlighted the value of CACS in risk stratification in hypertensive COVID-19 patients.

COVID-19 patients suffering from hypertension with moderate to severe coronary calcium scores tend to experience less favorable clinical outcomes [6, 12]. In particular, the mortality rate increased by $\sim 8 \%$ for every $100 \mathrm{~mm}^{3}$ increase in coronary calcium volume.

In fact, in multivariable Cox analysis, the coronary calcium score was a predictor of mortality, regardless of age, sex, creatinine, diabetes, and lung interstitial involvement [7, 13-15].

Cardiovascular calcifications are associated with nonphysiological aging and reflect atherosclerotic burden [8,9]. In addition, they are associated with arterial stiffening and are a leading cause of isolated systolic hypertension in the elderly population.

CACS can be easily measured from chest CT performed for pneumonia assessment [7, 9, 16-18], and CACS has been demonstrated to be a strong independent predictor of future cardiovascular events [16, 17].

We propose 3 hypotheses that explain the predictive ability of the coronary calcium score in COVID- 19 .

The first "cardiovascular" hypothesis is that CAC is a more sensitive cardiovascular marker than other anamnestic variables (probably weakened by suboptimal data collection in retrospective studies). CAC is a cumulative marker of cardiovascular damage with varying degrees of severity, even in asymptomatic patients.

The second hypothesis is that of "frailty". Patient with more cardiovascular calcifications and who are more fragile and elderly and experience complications of SARS-CoV2 disease have higher mortality.
Fig. 3 Mortality risk map for Covid-19 according to hypertensive state and severity of coronary calcifications

\begin{tabular}{|c|c|c|c|c|}
\hline \multicolumn{5}{|c|}{ Covid-19 Hospital Mortality Risk Card } \\
\cline { 2 - 5 } Mortality & CAC 0 & CAC 0-100 & CAC 100-400 & CAC > 400 \\
\hline No Hypertension & $1,6 \%$ & $1,3 \%$ & $1,2 \%$ & $3,3 \%$ \\
\hline $\begin{array}{l}\text { Hypertension } \\
\text { Risk of In-Hospital }\end{array}$ & $2,9 \%$ & $2,5 \%$ & $7,5 \%$ \\
\hline $\begin{array}{l}\text { Death }<2 \% \\
\text { Risk of In-Hospital } \\
\text { Death 2-5\% }\end{array}$ & $\begin{array}{l}\text { Risk of In-Hospital } \\
\text { Death }>5 \%\end{array}$ \\
\hline
\end{tabular}


Fig. 4 30-day survival curves based on hypertensive status and coronary calcification severity

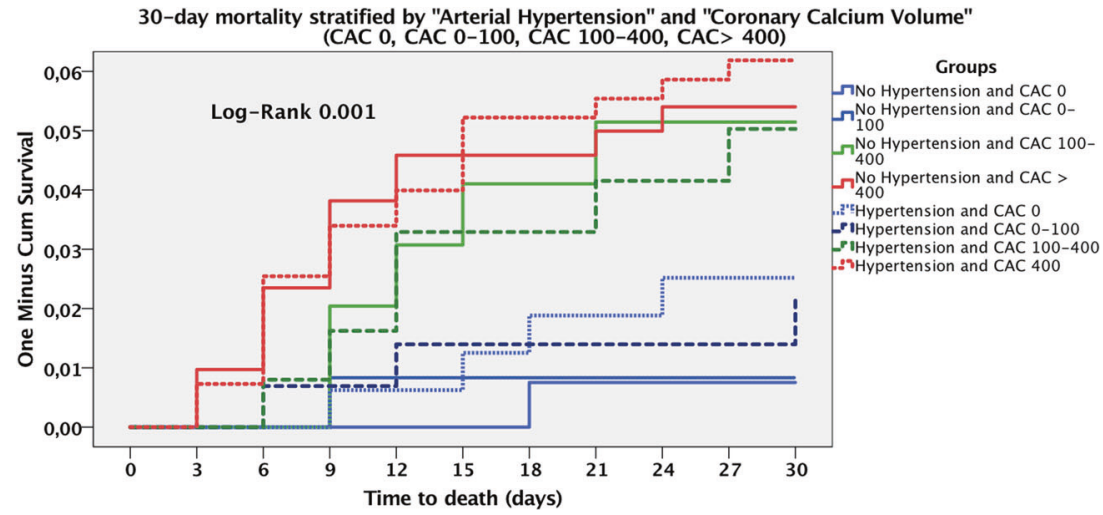

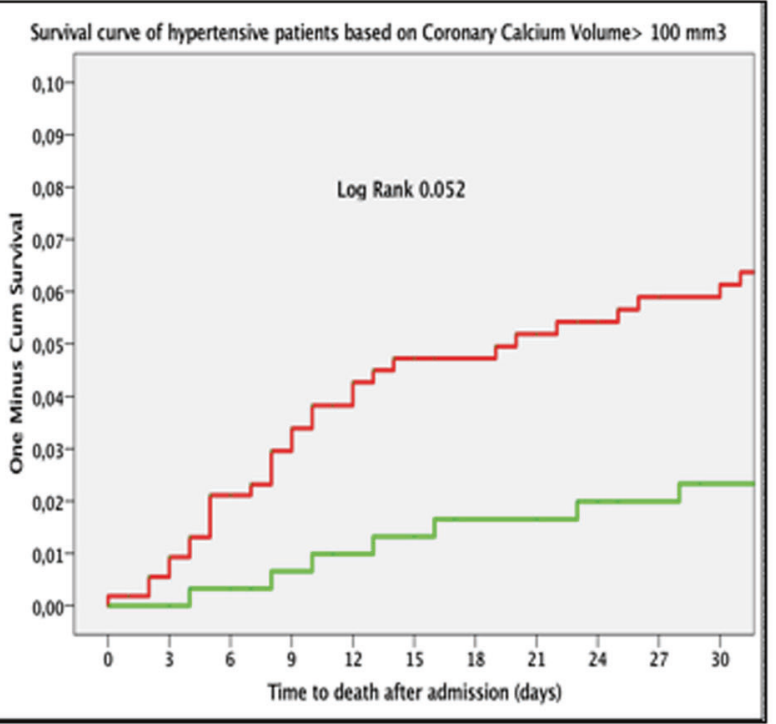

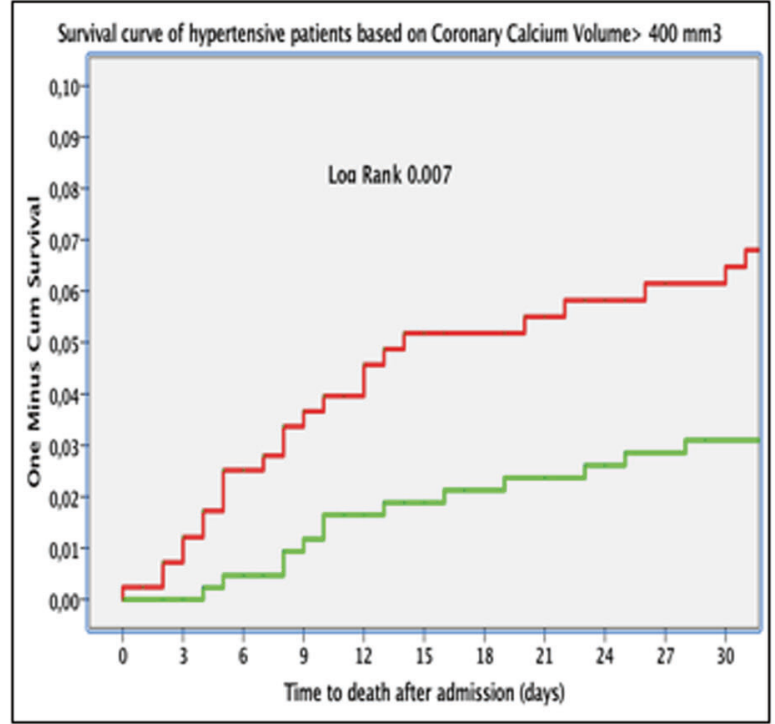

Fig. 5 Mortality of hypertensive patients based on CAC $100 \mathrm{~mm} 3$ and CAC $400 \mathrm{~mm} 3$ cut-off values

The third hypothesis is the "immunological" hypothesis; these patients have a higher baseline cardiometabolic inflammatory phenotype with a more pronounced inflammatory response that exposes them more to the SARS-CoV2-induced cytokine storm.

There is growing interest in using the CAC score to stratify the risk of hypertensive patients for the selection of personalized targets for blood pressure and cardiovascular prevention.

In the CAC Consortium study, in 6375 patients with "SPRINT-like" characteristics (age $>50$ years and Framingham risk score $>15 \%$ ), it was shown that CAC stratifies hypertension and recategorizes the 10-year risk probability of cardiovascular events [19].

In the same way, our results highlight the value of the calcium score in risk stratification in hypertensive COVID-19 patients, increasing the risk of adverse events and mortality.

Previous studies found that in COVID-19 patients, despite the presence of comorbidities, coronary calcium and its severity were associated with MACEs (acute coronary syndrome, pulmonary embolism, and stroke) and mortality $[7,9,20]$.

The coronary calcium score stratifies hypertensive patients, identifying clinical and subclinical atherosclerosis, heart damage and potentially increased susceptibility to disease progression and severity in hypertensive COVID-19 patients [10].

Hence, coronary calcium appears to be a promising biomarker in SARS-CoV-2 disease patients with prognostic significance [11].

Patients with a higher calcification burden have a worse baseline risk profile, as they have worse endothelial function, and hypoxic damage can exacerbate myocardial stress.

The vascular calcification burden is associated with a greater atherosclerotic burden, which confers greater susceptibility to more aggressive viral replication, greater inflammatory stimuli, and therefore more cardiovascular manifestations of COVID-19. 
Table 4 Univariate and multivariate Cox regression for Mortality

\begin{tabular}{|c|c|c|c|c|}
\hline \multirow[t]{3}{*}{ Variables } & \multicolumn{4}{|c|}{ Patients with hypertension } \\
\hline & \multirow[b]{2}{*}{$\mathrm{HR}$} & \multirow[b]{2}{*}{ Lower } & \multicolumn{2}{|c|}{$95 \%$ IC } \\
\hline & & & Upper & $P$ value \\
\hline \multicolumn{5}{|l|}{ Univariate cox regression for mortality } \\
\hline Age, years, \pm SD & 1,051 & 1,038 & 1,063 & 0.001 \\
\hline Female sex & 0,654 & 0,484 & 0,844 & 0.006 \\
\hline Diabetes & 1,391 & 1,056 & 1,831 & 0.019 \\
\hline Creatinine, $\mathrm{mg} / \mathrm{dl}$ & 1,366 & 1,247 & 1,496 & 0.001 \\
\hline Coronary calcium volume, $\mathrm{mm}^{3}$ & 1,001 & 1,000 & 1,001 & 0.003 \\
\hline Lung interstitial involvement, $n / N$ & 1,2 & 1,034 & 1,408 & 0.017 \\
\hline \multicolumn{5}{|c|}{ Multivariate cox regression for mortality (coronary calcium volume) } \\
\hline Age, years, \pm SD & 1,045 & 1,032 & 1,058 & 0.001 \\
\hline Female sex & 0,695 & 0,504 & 0,958 & 0.026 \\
\hline Diabetes & 1,289 & 0,973 & 1,707 & 0.077 \\
\hline Creatinine, $\mathrm{mg} / \mathrm{dl}$ & 1,239 & 1,121 & 1,370 & 0.001 \\
\hline Coronary calcium volume, $\mathrm{mm}^{3}$ & 1,001 & 1,000 & 1,001 & 0.001 \\
\hline Lung interstitial involvement, $n / N$ & 1,216 & 1,037 & 1,425 & 0.016 \\
\hline \multicolumn{5}{|c|}{ Multivariate cox regression for mortality (coronary calcium volume > 100) } \\
\hline Age, years, \pm SD & 1,048 & 1,035 & 1,061 & 0.001 \\
\hline Female sex & 0,705 & 0,511 & 0,972 & 0.033 \\
\hline Diabetes & 1,296 & 0,978 & 1,717 & 0.071 \\
\hline Creatinine, $\mathrm{mg} / \mathrm{dl}$ & 1,231 & 1,114 & 1,361 & 0.001 \\
\hline Coronary calcium volume $>100, \mathrm{~mm}^{3}$ & 1,345 & 1,029 & 1,757 & 0.030 \\
\hline Lung interstitial involvement, $n / N$ & 1,204 & 1,027 & 1,411 & 0.022 \\
\hline \multicolumn{5}{|c|}{ Multivariate cox regression for mortality (coronary calcium volume $>400$ ) } \\
\hline Age, years, \pm SD & 1,047 & 1,034 & 1,060 & 0.001 \\
\hline Female sex & 0,698 & 0,506 & 0,962 & 0.028 \\
\hline Diabetes & 1,280 & 0,966 & 1,696 & 0.085 \\
\hline Creatinine, $\mathrm{mg} / \mathrm{dl}$ & 1,242 & 1,123 & 1,373 & 0.001 \\
\hline Coronary calcium volume $>400, \mathrm{~mm}^{3}$ & 1,315 & 1,010 & 1,713 & 0.042 \\
\hline Lung interstitial involvement, $n / N$ & 1,214 & 1,036 & 1,422 & 0.016 \\
\hline \multicolumn{5}{|c|}{$\begin{array}{l}\text { Multivariate cox regression for mortality (coronary calcium volume ordinal } \\
\text { variabile for every } 100 \mathrm{~mm}^{3} \text { ) }\end{array}$} \\
\hline Age, years, \pm SD & 1,044 & 1,031 & 1,058 & 0.001 \\
\hline Female sex & 0,708 & 0,512 & 0,981 & 0.038 \\
\hline Diabetes & 1,286 & 0,966 & 1,713 & 0.085 \\
\hline Creatinine, $\mathrm{mg} / \mathrm{dl}$ & 1,235 & 1,117 & 1,366 & 0.001 \\
\hline Coronary calcium volume, $\mathrm{mm}^{3}$ & 1,082 & 1,033 & 1,133 & 0.001 \\
\hline Lung interstitial involvement, $n / N$ & 1,200 & 1,020 & 1,412 & 0.028 \\
\hline
\end{tabular}

Cox regression models: top to bottom univariate Cox regression, multivariate Cox regression with quantitative coronary calcifications (volume $\mathrm{mm}^{3}$ ), multivariate cox regression using coronary calcifications categorized according to cut-offs of 100 and $400 \mathrm{~mm}^{3}$. In the last model, calcium volume is expressed as an ordinal variable for every $100 \mathrm{~mm}^{3}$ of calcium volume (including only patients with values between 0 and $1000 \mathrm{~mm}^{3}$ )

In a disease with cardiovascular tropism, cardiovascular calcifications could indirectly represent the cardiovascular health of an individual (independent of other anamnestic and laboratory variables), and their quantification allows evaluation of cardiovascular resilience to SARS-CoV-2.

The burden of cardiovascular calcifications probably reflects presumed greater endothelial dysfunction. Patients at high cardiovascular risk (identified by the greater burden of calcifications) are more susceptible to viral endothelial damage and thus develop a more aggressive disease.

Whether the calcification burden correlates with a different inflammatory response remains to be evaluated in the future.

The correlation between coronary artery calcification and epicardial adipose tissue (EAT) is known in the literature and includes both atherosclerotic and cardiometabolic risks. Epicardial adipose tissue inflammation was found to be associated with troponin increases in COVID-19 patients and more severe disease.

In Slipczuk's work [10], the combination of CAC and EAT further stratifies composite outcomes in the COVID19 population.

The values of EAT appear higher than to those of CAC in terms of stratification, suggesting a different inflammatory response phenotype (TNF and IL-6) that favors the cytokine storm, which is related to severe cases of COVID-19.

The coronary calcium score in COVID-19 proved to be a versatile marker that is easy to use for both diagnosis and prognostic assessment [21, 22].

Its ease of use may add value and retrospectively complement published data on hypertension in COVID-19 patients.

From the data of pharmacological trials, it can be inferred whether patients with coronary calcifications respond better or worse to certain treatments (primarily heparin and steroids), indicating this imaging biomarker's “diagnostic and therapeutic" value.

From the perspective of hypertensive patients, it will be interesting to verify the correlation of the coronary calcium score with other markers of subclinical organ damage (cardiac, carotid, renal, peripheral arterial) and understand the real potential of this imaging biomarker [23-25].

\section{Limitations of the study}

The results of the study must be considered in the context of some methodological limitations and biases.

First, the data from our work refer to the first pandemic wave in the Western world.

Within this very short time, the country has been hit by nothing short of a tsunami of unprecedented force, punctuated by an incessant stream of deaths.

Italy appears to be the country with the highest proportion of severely ill patients requiring invasive ventilation and with the highest percentage of deaths, with the health care system being heavily burdened by the strain of the current epidemic.

In particular, the first pandemic wave was characterized by a lack of RNA-PCR nasopharyngeal swabs (which justified extensive use of chest $\mathrm{CT}$ at hospital admission) and a 
lack of health care organization and ICU beds. The lack of ICU beds and personnel trained in invasive/noninvasive ventilation techniques may have affected respiratory outcomes.

Another important bias common in the COVID-19 literature is the lack of prespecified causes of death in patients with SARS-CoV-2 infection. COVID-19-related deaths are not clearly defined in the international reports available thus far, and differences in definitions of what is or is not a COVID-19-related death might explain variation in casefatality rates among different countries.

Furthermore, we have no information regarding the empirical therapies used to treat these patients since evidence on heparin, corticosteroids and ventilatory therapy emerged in the subsequent pandemic waves.

Even if the definition of hypertension is validated by administrative data (and is therefore reliable), we have no information regarding the classes of antihypertensives taken by these patients.

\section{Future research perspectives}

The coronary calcium score promises to be an ideal marker, as it correlates with COVID-19 clinical outcomes, is both a qualitative and quantitative marker, is not costly, and provides additional information.

The coronary calcium score can be understood as a surrogate marker of cardiovascular disease and certainly deserves further study and investigation.

The current advantage of the calcium score is that it can be calculated retrospectively and better clarify the relationships between hypertension, cardiovascular disease, and COVID-19 outcomes.

Cardiovascular calcifications could clarify, both retrospectively and prospectively, some aspects of cardiovascular pathophysiology still not fully elucidated in the context of SARS-CoV-2 infection.

By conducting a subanalysis of the randomized clinical trials that performed chest $\mathrm{CT}$ as criteria for inclusion in the study, it will be possible to know whether patients with coronary calcifications respond better or worse to available pharmacological and respiratory therapies.

\section{Conclusions}

The coronary calcium score contributes to the stratification of the risk of adverse outcomes (especially mortality) in hypertensive COVID-19 patients.

The classification of COVID-19 patients based on hypertensive status and the severity of coronary calcifications allows the identification of subgroups at greater risk of respiratory support, cardiovascular events (ACS, stroke, and pulmonary embolism), and mortality.

The coronary calcium score, regardless of age, sex, creatinine, diabetes, and interstitial lung involvement, predicted the risk of death in hypertensive patients.

Coronary calcification burden (in terms of volume $\mathrm{mm}^{3}$ ) was associated with an increased risk of death regardless of age, sex, creatinine, diabetes, and lung interstitial involvement. Cardiovascular calcifications appear to be a promising imaging marker that can provide pathophysiological insight into cardiovascular risk factors and COVID-19 outcomes.

\section{Compliance with ethical standards}

Conflict of interest The authors declare no competing interests.

Publisher's note Springer Nature remains neutral with regard to jurisdictional claims in published maps and institutional affiliations.

\section{References}

1. Wang FM, Rozanski A, Arnson Y, Budoff MJ, Miedema MD, Nasir K. et al. Cardiovascular and all-cause mortality risk by coronary artery calcium scores and percentiles among older adult males and females. Am J Med. 2021;134:341-50.e1.

2. Williams B, Mancia G, Spiering W, Agabiti Rosei E, Azizi M, Burnier M.ESC Scientific Document Group et al. 2018 ESC/ESH Guidelines for the management of arterial hypertension. Eur Heart J. 2018;39:3021-104.

3. Mancia G, Rea F, Ludergnani M, Apolone G, Corrao G. Reninangiotensin-aldosterone system blockers and the risk of Covid-19. N. Engl J Med. 2020;382:2431-40.

4. Grasselli G, Greco M, Zanella A, Albano G, Antonelli M, Bellani G. et al. COVID-19 Lombardy ICU Network. Risk factors associated with mortality among patients with COVID-19 in intensive care units in Lombardy. Italy JAMA Intern Med. 2020;180: $1345-55$.

5. De Simone G. Position Statement of the ESC Council on Hypertension on ACE-Inhibitors and Angiotensin Receptor Blockers. European Society of Cardiology $>$ Councils $>$ Council on Hypertension > News 2020 Mar 2020.

6. Colombi D, Villani GD, Maffi G, Risoli C, Bodini FC, Petrini M. et al. Qualitative and quantitative chest CT parameters as predictors of specific mortality in COVID-19 patients. Emerg Radio. 2020;27:701-10.

7. Giannini F, Toselli M, Palmisano A, Cereda A, Vignale D, Leone R. et al. Coronary and total thoracic calcium scores predict mortality and provides pathophysiologic insights in COVID-19 patients. J Cardiovasc Comput Tomogr. 2021;15:421-30.

8. Esposito A, Palmisano A, Toselli M, Vignale D, Cereda A, Rancoita PMV. et al. Chest CT-derived pulmonary artery enlargement at the admission predicts overall survival in COVID-19 patients: insight from 1461 consecutive patients in Italy. Eur Radio. 2021;31:4031-41.

9. Sticchi A, Cereda A, Toselli M, Esposito A, Palmisano A, Vignale D. et al. Diabetes and mortality in patients with COVID-19: are we missing the link?. Anatol J Cardiol. 2021;25:376-9.

10. Zimmermann GS, Fingerle AA, Müller-Leisse C, Gassert F, von Schacky CE, Ibrahim T. et al. Coronary calcium scoring assessed on native screening chest $\mathrm{CT}$ imaging as predictor for outcome in 
COVID-19: an analysis of a hospitalized German cohort. PLoS ONE. 2020;15:e0244707.

11. Cereda A, Toselli M, Palmisano A, Leone R, Vignale D, Nicoletti V, et al. Is pleural effusion in COVID-19 interstitial pneumonia related to in-hospital mortality? Italian Journal of Medicine, v. 15, 2021.

12. Cosyns B, Motoc A, Luchian ML, Lochy S, Belsack D. Coronary calcium score in COVID-19 hospitalized patients. JACC Cardiovasc Imaging. 2020;13:2698.

13. Dillinger JG, Benmessaoud FA, Pezel T, Voicu S, Sideris G, Chergui N. et al. COVID Research Group of Lariboisiere Hospital. Coronary artery calcification and complications in patients with COVID-19. JACC Cardiovasc Imaging. 2020;13:2468-70.

14. Fervers P, Kottlors J, Zopfs D, Bremm J, Maintz D, Safarov O. et al. Calcification of the thoracic aorta on low-dose chest CT predicts severe COVID-19. PLoS ONE. 2020;15:e0244267.

15. Fovino LN, Cademartiri F, Tarantini G. Subclinical coronary artery disease in COVID-19 patients. Eur. Heart J Cardiovasc Imaging.2020;21:1055-6.

16. Gupta YS, Finkelstein M, Manna S, Toussie D, Bernheim A, Little BP. et al. Coronary artery calcification in COVID-19 patients: an imaging biomarker for adverse clinical outcomes. Clin Imaging. 2021;77:1-8.

17. Nair AV, Kumar D, Yadav SK, Nepal P, Jacob B, Al-Heidous M. Utility of visual coronary artery calcification on non-cardiac gated thoracic CT in predicting clinical severity and outcome in COVID-19. Clin Imaging. 2021;74:123-30.

18. Shabbir MA, Koutroumpakis E, Powell C, Fatakdawala M, Lin K, Taegtmeyer $\mathrm{H}$, et al. Coronary artery calcification heralds adverse clinical outcomes in patients hospitalized for covid-19. J Am Coll Cardiol. 2021;77:3065.

19. Adelhoefer S, Uddin SMI, Osei AD, Obisesan OH, Blaha MJ, Dzaye O. Coronary artery calcium scoring: new insights into clinical interpretation-lessons from the CAC Consortium. Radio Cardiothorac Imaging. 2020;2:e200281.

20. Slipczuk L, Castagna F, Schonberger A, Novogrodsky E, Sekerak $\mathrm{R}$, Dey D. et al. Coronary artery calcification and epicardial adipose tissue as independent predictors of mortality in COVID-19. Int J Cardiovasc Imaging. 2021;37:3093-100.

21. Cereda A, Toselli M, Laricchia A, Mangieri A, Ruggiero R, Gallo F, et al. Stress-induced cardiomyopathy related to SARS-CoV-2. Heart Mind. 2020;4:57-8.

22. Scoccia A, Gallone G, Cereda A, Palmisano A, Vignale D, Leone R. et al. Impact of clinical and subclinical coronary artery disease as assessed by coronary artery calcium in COVID-19. Atherosclerosis. 2021;328:136-43.

23. Ahmed AI, Raggi P, Al-Mallah MH. Teaching an old dog new tricks: The prognostic role of CACS in hospitalized COVID-19 patients. Atherosclerosis. 2021;S0021-9150:00251-3.

24. Nishiga M, Wang DW, Han Y, Lewis DB, Wu JC. COVID-19 and cardiovascular disease: from basic mechanisms to clinical perspectives. Nat Rev Cardiol. 2020;17:543-58. https://doi.org/ 10.1038/s41569-020-0413-9.

25. Cereda A, Toselli M, Palmisano A, Vignale D, Leone R, Nicoletti $\mathrm{V}$, et al. The hidden interplay between sex and COVID-19 mortality: the role of cardiovascular calcification. Geroscience. 2021;14:1-15. 